\author{
Military Technical College \\ Kobry El-Kobbah, \\ Cairo, Egypt.
}

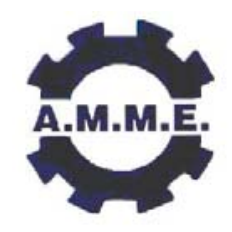

\title{
EFFECTS OF OSCILLATORY PLUNGING MOTION ON ROTARY WING BLADES
}

\author{
ABOURAHMA* A.A.
}

\begin{abstract}
The effect of blade oscillation on resulting air loads has promoted new interest in oscillatory blade aerodynamics. Rotary wings aerodynamics is considerably more complex than fixed wing aerodynamics. As an airfoil performs an oscillation, vortices are shed into the medium with a circulation strength equal in magnitude to the increase in circulation about the airfoil but opposite in direction. These disturbances are stored in the fluid because the shed vorticity convicts down stream at a local flow field velocity. The wake of the rotor consists of helical vortex sheet below the rotor b1ades. The wake remains near the rotor and therefore passes close to the following blades.
\end{abstract}

The fundamental closed form solution of Theodorsen [1], and Lowey [2], provide the basis for theoretical work in this area. The closed form theory shows rapid changes in the lift deficiency function with changes in reduced frequency, wake spacing and frequency ratio. In the past, emphasis in the study of unsteady aerodynamics has tended to focus on flutter instability and the effect of unsteady aerodynamics on generating lift and torsional loads. The classic reference on this subject is that by Garrick [3]. While Garrick's work shows that an airfoil oscillating in pitch will typically produce drag in the lower reduced frequency range, $k$, it is found that the presence of another layer of shed vorticity of the proper phase can reduce the drag on plunging airfoils and depending upon wake spacing, reduced frequency and phase, may even enhance the propulsive force acting on the plunging airfoils.

\section{KEY WORDS}

Unsteady Aerodynamics, Oscillatory Plunging Motion and rotary wing aerodynamics.

\footnotetext{
* Ph. D Head of ARC Engineering Department. Cairo, Egypt.
} 


\section{HIGHER HARMONIC CONTROL (HHC)}

$\mathrm{HHC}$ is an electronic, computer-controlled active vibration suppression system which senses and cancels vibrations in a helicopter by $\mathrm{N} /$ revolution feathering or pitch motion of the rotor blades, $\mathrm{N}$ being the number of blades. Higher Harmonic Control is an active helicopter vibration control concept which alters the aerodynamic loads on the rotor blades such that the blade response is reduced. This in turn reduces the vibratory forces and moments acting at the hub, which cause airframe vibration. When the drag force is reversed, acting to propel the airfoil forward, it is generally referred to as either negative drag or propulsive force.

\section{NASA-ARMY OH-6A FLIGHT TEST PROGRAM}

Open loop performance data from the NASA-Army OH-6A higher harmonic control (HHC) flight test program, Wood et al. [4], show significant reductions in main rotor shaft torque and engine power in the airspeed regime from hover to 100 knots. Depending upon $\mathrm{HHC}$ controller phase and helicopter airspeed, reductions in power were recorded as large as $20 \%$, with reductions of the order of $10 \%$ being typical.

\section{ADDITION OF WAKE EFFECTS}

Here, the emphasis will be in the study of the effects of oscillating plunging motion drag reduction on rotary wing blades. The panel method developed by Platzer et al. [5], was applied for unsteady incompressible flow past airfoils or airfoil combinations of arbitrary geometry and amplitudes of different kinds of motions. The specific mechanisms, that have been used in this research to discuss the phenomenon of $\mathrm{OH}-6 \mathrm{~A}$ helicopter rotor power reduction, are: (1) the unsteady aerodynamics associated with a plunging airfoil and how that produces a propulsive force or decrease in rotor power; (2) the influence of a layer of wake vorticity on this propulsive force, which represents the influence of the unsteady aerodynamics of one rotor blade on adjacent blades, and (3) the influence of the phasing of a layer of shed vorticity on this drag force, and how, depending upon specific phasing, the effect on the airfoil will be either drag or propulsive force [6].

The span-wise variation of bound circulation results in trailed vorticity parallel to the free stream direction. Time variation of the bound circulation leads to shed vorticity parallel to the wingspan. The wake is composed of sheets of vorticity convicted downstream from the trailing edge by the free stream velocity. The wake of a rotor in hover or in vertical flight consists of helical vortex sheets below the disk, one from each blade. Unsteady motion of the rotor blade will produce a shed vorticity in the wake spirals. With low disk loading (at low collective pitch settings), the wake remains near the rotor disk and therefore passes close to the following blades. Thus the wake vorticity is not convicted down stream of the airfoil as with a fixed wing.

For a helicopter in hover, the rotation of the wing introduces a number of features that require special attention, notably the returning vortex wake, the time varying free stream, radial flow, and a fundamentally transcendental geometry that requires either approximate or numerical solutions. The lift on a wing is due to its bound circulation. Conservation of vorticity in the flow requires that there be trailed and shed vorticity in the wake behind the wing. For high inflow (high collective pitch settings) or in high- 
speed forward flight, the wake will be convicted away from the blades. The returning shed wake influence is primarily a concern of hover and vertical flight, and forward flight at airspeeds less than 100 knots. Assuming high aspect ratio of the blade, lifting line theory requires knowledge of the loads on the blade section, and the returning shed wake of the rotor must be incorporated into the two-dimensional unsteady airfoil theory.

The wake in hover or vertical flight consists of helical vortex sheets below the disk, one from each blade. Unsteady motion of the rotor blade will produce shed vorticity in the wake spirals. Figures (1A) through (1D), from Loewy [4], illustrate the basic elements of the unsteady flow field of a helicopter rotor. A rotor in hovering or vertical flight produces a trailing tip vortex with a downward axial velocity that, if otherwise undisturbed, forms a contracting helix as shown in Figure $(1 \mathrm{~A})$. If the inflow over the rotor disk is constant, then the fluid off the trailing edge of the blades makes a helical surface with horizontal radial elements as shown in Figure $(1 \mathrm{~B})$. If there is an oscillation in blade effective angle of attack, blade lift will alternate also, and as a result of these changes in lift, vortices will be shed continuously at the blade trailing edge.

These vortices fall along the horizontal radial elements of the helical surface shown in Figure $(1 \mathrm{~B})$, so long as the oscillations in angle of attack are small, Figure (1C) illustrates this local sheet of shed vorticity. It should be noted that vorticity is considered to be on the helical surface shown in Figure (1B); the vertical displacements from that surface shown in Figure (1C) represent the strength of the vorticity at a particular azimuthal and radial position.

The variation in this vertical displacement (hence vortex strength) around the azimuth corresponds to the history of the motion of a given blade element at a fixed radius. Variation of shed vortex strength in the radial direction at any fixed azimuth angle is a function of the variation with blade span of: (I) blade plan form, (2) amplitude of oscillation of effective angle of attack, and (3) relative air velocity. Since, from the Helmholtz theorem, vorticity cannot begin or end in space, a variation of shed vorticity in the radial direction implies that there are trailing vortices at constant radii similar to and inboard of the tip vortex. These trailing vortices have been induced in Figure (1D). The schematic drawings, Figures (1A) through (1D), indicate the difficulty of attempting to obtain a complete representation of unsteady rotor aerodynamics. One means of simplifying the problem is to consider extreme "pitch" values for the helix. For the case of very high inflow velocity, $\mathrm{u}$, in relation to $\mathrm{n}$, and the opposite, where $\mathrm{u}$ is very low compared to $\Omega$. This is shown in Figure (2).

When the vertical spacing between adjacent helical surfaces of shed vorticity is very large, then we expect that all shed vorticity beyond a small fraction of a revolution would be too far below the blade in question to have a significant effect. Under these conditions, it would be sufficient to account for only the attached vortex sheet within that fraction of a revolution, as in Figure (2 A).

On the other hand, when the vertical spacing between the adjacent helical surfaces of shed vorticity is very small, all the sheets of shed vorticity tend to pile up on each other, and the effect of that vorticity close to the blade in question (shed by blade passes) is of more importance than that which exists beyond a reference azimuth angle on either side of the blade. This situation is depicted in Figure (2B), Ref. [4].

Since the shed vorticity is being accounted for as perturbation in the main flow, this 
component of induced velocity will be small compared to the free-stream velocity (except when the spacing approaches zero) and must react with small, first order airfoil displacements.

Because of the physical unrealities associated with the standard mathematical model representation of the problem (such as zero thickness of wake and airfoil, zero displacement of the wake from horizontal planes, and infinite velocities at the core of a vortex), the accuracy of results as the wake spacing goes to zero is questionable. The effect of the horizontal component of induced flow, therefore, has been neglected in Loewy's analysis. Shown in Figure (3) is a two-dimensional model of unsteady rotor aerodynamics for a single-bladed rotor operating at low inflow [6]. With an arrow indicating that the rows of vorticity under the rotor disc extend to infinity where $n$, number of revolution index, $q$ is the number of blades index, $Q$ is the total number of blades and $\mathrm{h}$ is the vertical distance between successive rows of vorticity. Consistent with the idea that only the vorticity near the blade section has an important effect, one may allow the planar rows of vorticity to extend to infinity in the horizontal direction in order to achieve mathematical simplification.

\section{UNSTEADY PANEL CODE}

The extension of this method to an airfoil experiencing a rapid change in angle of attack can be accomplished as follows. It is important to recall Prandtl's early flow visualization experiments which demonstrated the generation of a starting vortex off the trailing-edge whenever the angle of attack was changed. Hence continuous changes in angle of attack produce a continuous shedding of vorticity into the trailing wake. This shedding can be explained by the Helmholtz vortex theorem which requires that any change in the circulation around the airfoil must be matched by the appearance of an equal counter vortex at the trailing edge in order to achieve constancy of the total circulation in the flow field.

The vortices shed from the trailing edge move with the fluid particles of the surrounding fluid and hence are swept downstream with a speed essentially equal to the freestream speed. Therefore these vortices will stay close enough to the airfoil for a finite time to influence its pressure distribution. Any unsteady airfoil theory therefore must describe this vortex shedding process. It is this feature which distinguishes unsteady airfoil theory from its steady counterpart [5]. The above considerations suggest that the airfoil can be modeled by similar source and vortex distributions as in the steady case.

\section{DISCUSSION OF RESULTS}

In the present work, we are interested in the prediction of the thrust and drag forces generated by an oscillating airfoil. Calculations were performed to ascertain the panel code's capability. Figure (4) shows the comparison between Garrick's predictions and the panel code results [6]. These figures present plots of the average propulsive force coefficient vs the non-dimensional plunging amplitude of oscillation for a reduced frequencies, $k=0.1$. The two methods are in good agreement for small plunge amplitudes. As might be expected, the agreement starts to deteriorate with increasing plunge amplitude. The panel code was applied to an NACA 0009 airfoil using 100 panels. 
In figure (5), the wake patterns of two airfoils plunging in oscillatory motion is shown for zero wake phasing and a reduced frequency 0.0617 for the two airfoils. Figure (6) presents the drag coefficients vs. non dimensional time of the two airfoils shown in Figure (5). In figure (7), propulsive drag coefficient versus wake phasing from single airfoil [3], Single airfoil Panel Code, two airfoils (Loewy) [2], Two Airfoils Panel Code. [6]

\section{CONCLUSIONS}

The most important conclusions that can be drawn from this research are:

1. For drag, in pure plunge, wake phasing can increase or decrease the steady component of drag acting on the airfoil. The increase in the steady component of propulsive force due to wake phasing in the case of pure plunge was sufficient to significantly enhance the" Katzmayr" effect value of propulsive force.

2. For an airfoil oscillating in plunge, for optimum reduction in drag, the phase angle of the wake vorticity in the single wake case with respect to motion of the reference airfoil should be about $180^{\circ}$.

3. For an airfoil oscillating in plunge, the largest value of steady drag when the phase angle of the wake vorticity with respect to motion of the reference airfoil is about $0^{\circ}$.

4. There exists a phase angle, $75^{\circ}$ for the case, (reduced $k=.0617$, wake nondimensional spacing, $h=2$ ), where the effect of wake vorticity on the reference airfoil is identical to the case of the wake at infinity (represented in the code by wake $h=200$ ), or no wake vorticity at all. Applying these findings to the $\mathrm{OH}-6 \mathrm{~A}$ case, we can add the following conclusions :

a) Measured reductions in power are feasible when the "Katzmayr" effect and wake enhancements are included.

b) Measured higher harmonic control results show least benefit at 100 knots due to the wake being transported greatest distance from rotor at this speed [7].

\section{REFERENCES}

1. Theodorsen, T. "General Theory of Aerodynamic Instability and the Mechanism of flutter.", NACA T.R. 496, 1935.

2. Loewy, R. G., " A Two-Dimensional Approximation to the Unsteady Aerodynamics of Rotary Wings.", Journal of Aeronautical Science, Vol. 24, No. 2, Feb. 1957.

3. Garrick, I. E., "Propulsion of flapping and Oscillating aiafoil." NACA T. R. 567, 1936.

4. E. R. Wood, R. W. Powers, J. H. Clinc, and C. E. Hammand " On Developing and Flight Testing a Higher Harmonic Control System.", Journal of AHS, January, 1985.

5. Platzcr, M. F., Neace, K S., And Pang, C. K, Aerodynamic Analysis of Flapping Wing 
Propulsion", AIAA paper No. 93-0484, 31st Aerospace Science Meeting, Reno, NV, January 11-14, 1993.

6. Ahmed A. Abourahma, "The Wake Induced Unsteady Aerodynamic as Related to Higher Harmonic Control.", Ph. D. Dissertation, Naval Postgraduate School, Monterey, California 93943-5000, December, 1993.

7. E. R. Wood, Max F. Plalzer, Ahmed Ahourahma, Mark A. Coueh " On the Unsteady Aerodynamics of Higher Harmonic Control", Paper No. C 17, Nineteenth European Rotorcraft Forum, Cernohbio(Come), Italy September 1991. 

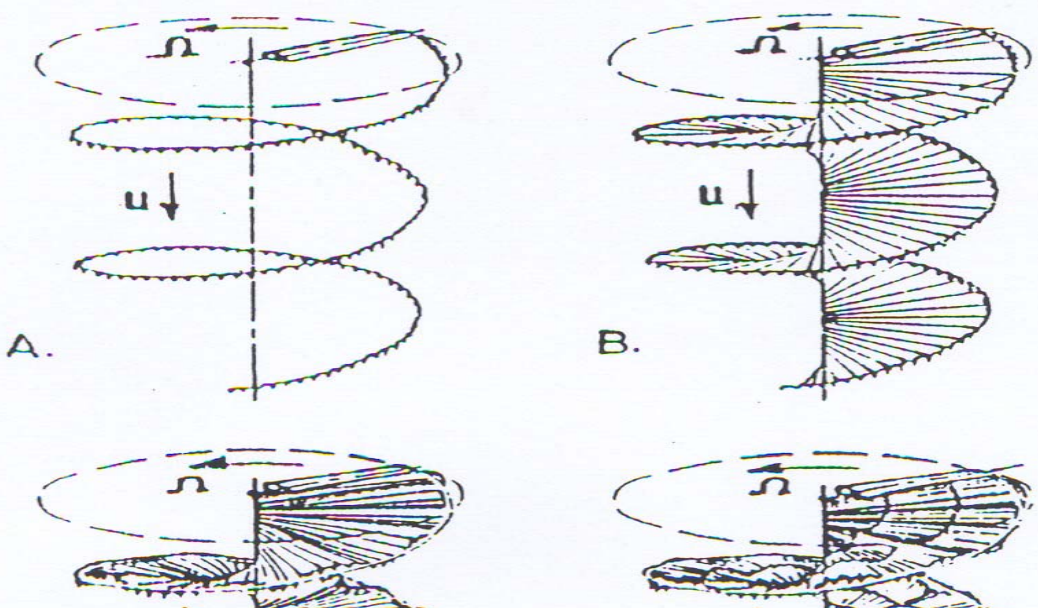

$4+$

$u \downarrow$
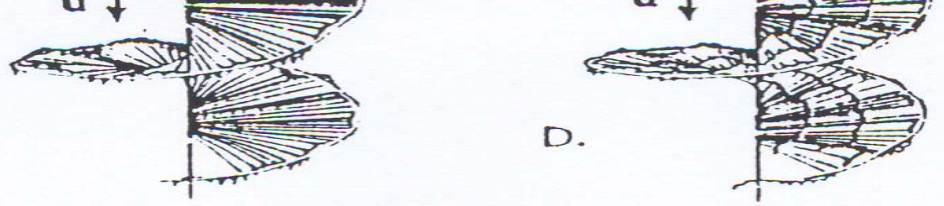

Fig.1 Schematic elements of unsteady rotor flow field
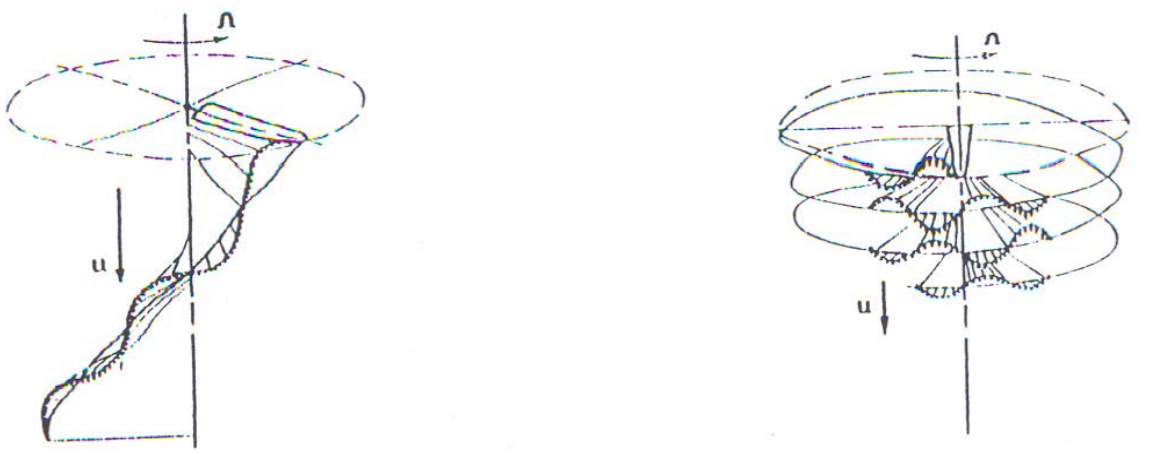

A. High Inflow

B. Low Inflow

Fig.2 Low and high inflow 


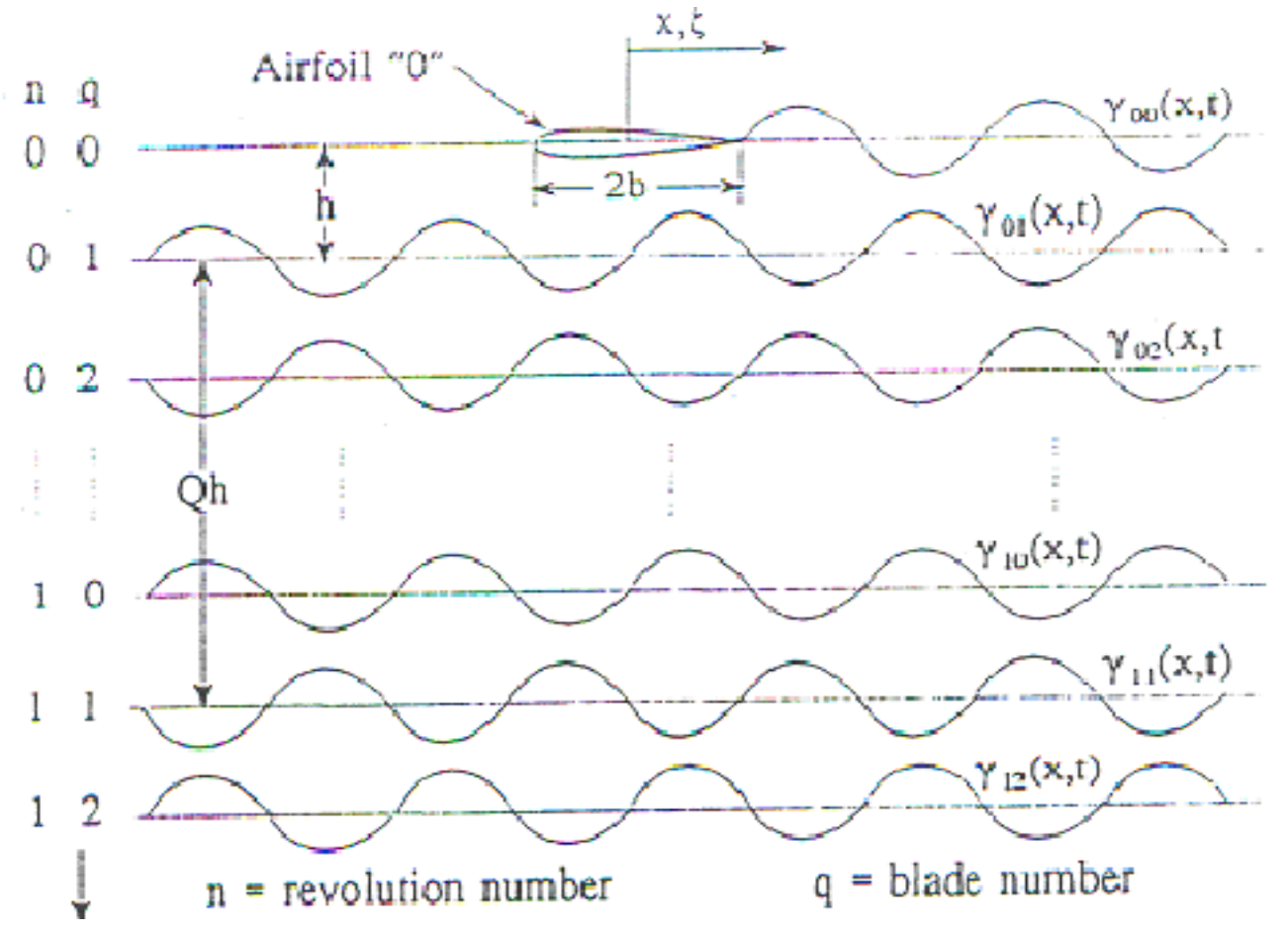

Fig.3 Two-dimensional model of unsteady rotor flow

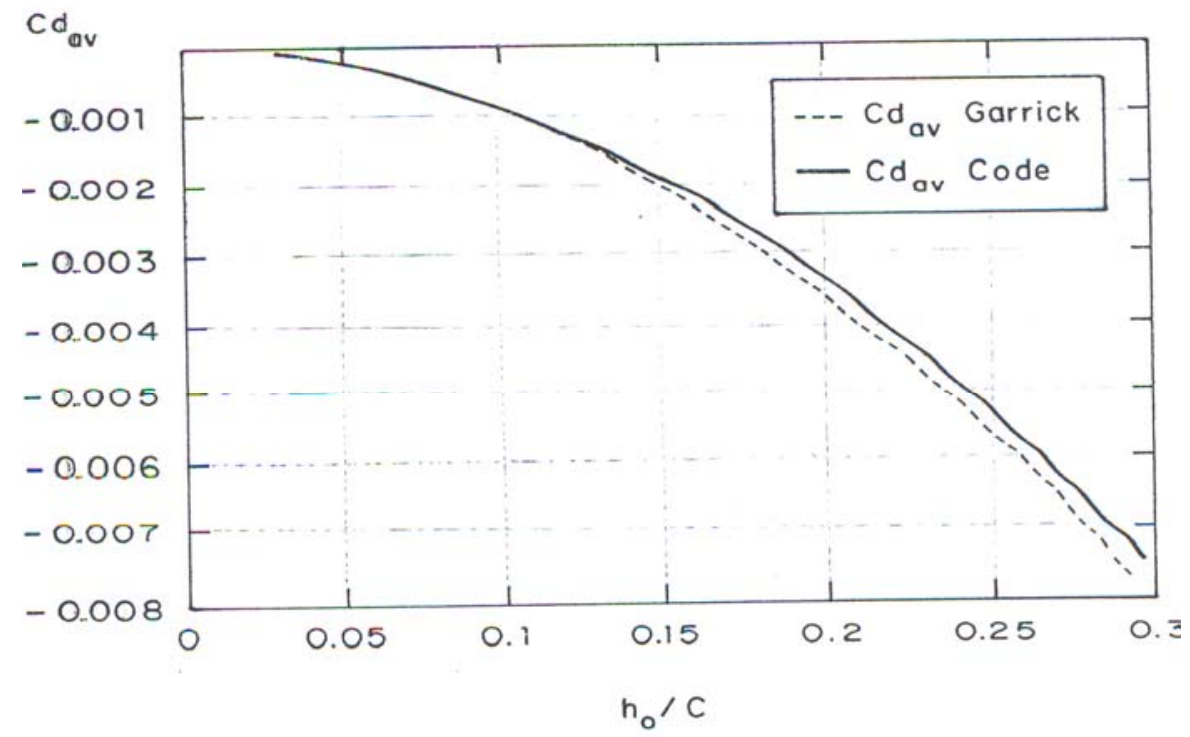

Fig. 4 Comparison of panel code results with Garrick. 


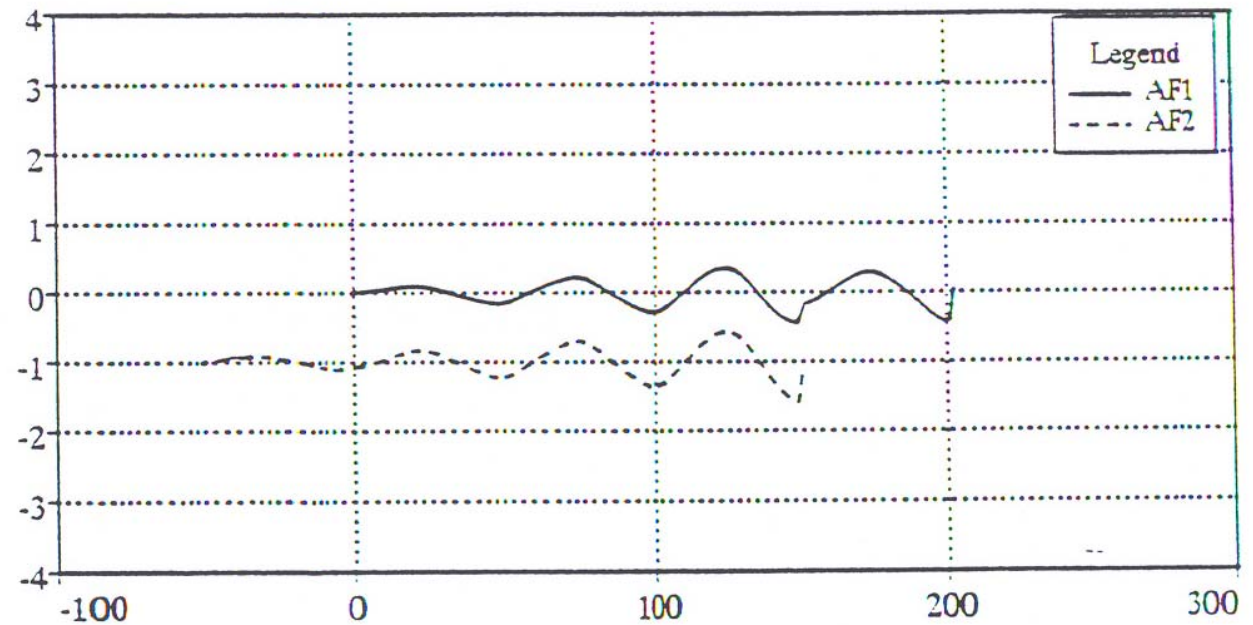

Fig. 5 Two airfoils in plunging oscillatory motion with zero wake phasing at $k=0.0617$

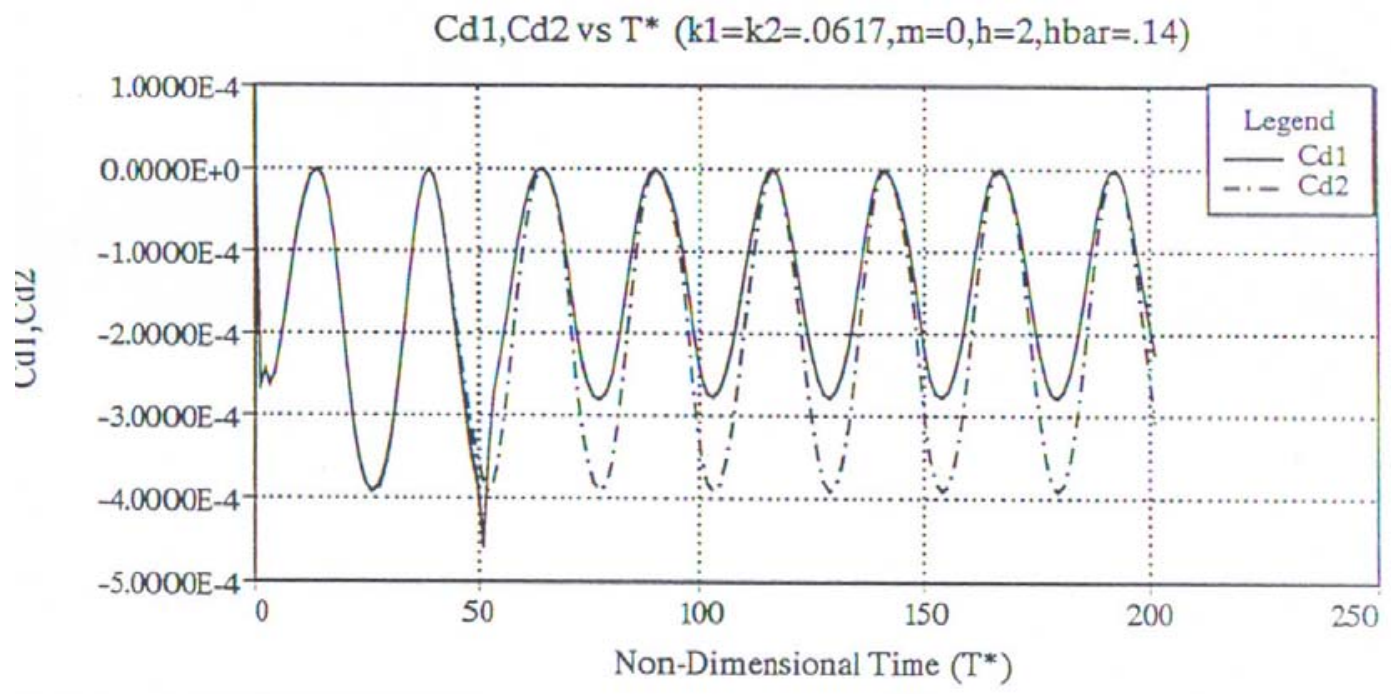

Fig. 6 Two airfoils in plunging oscillatory motion with zero wake phasing at $k=0.0617$ 


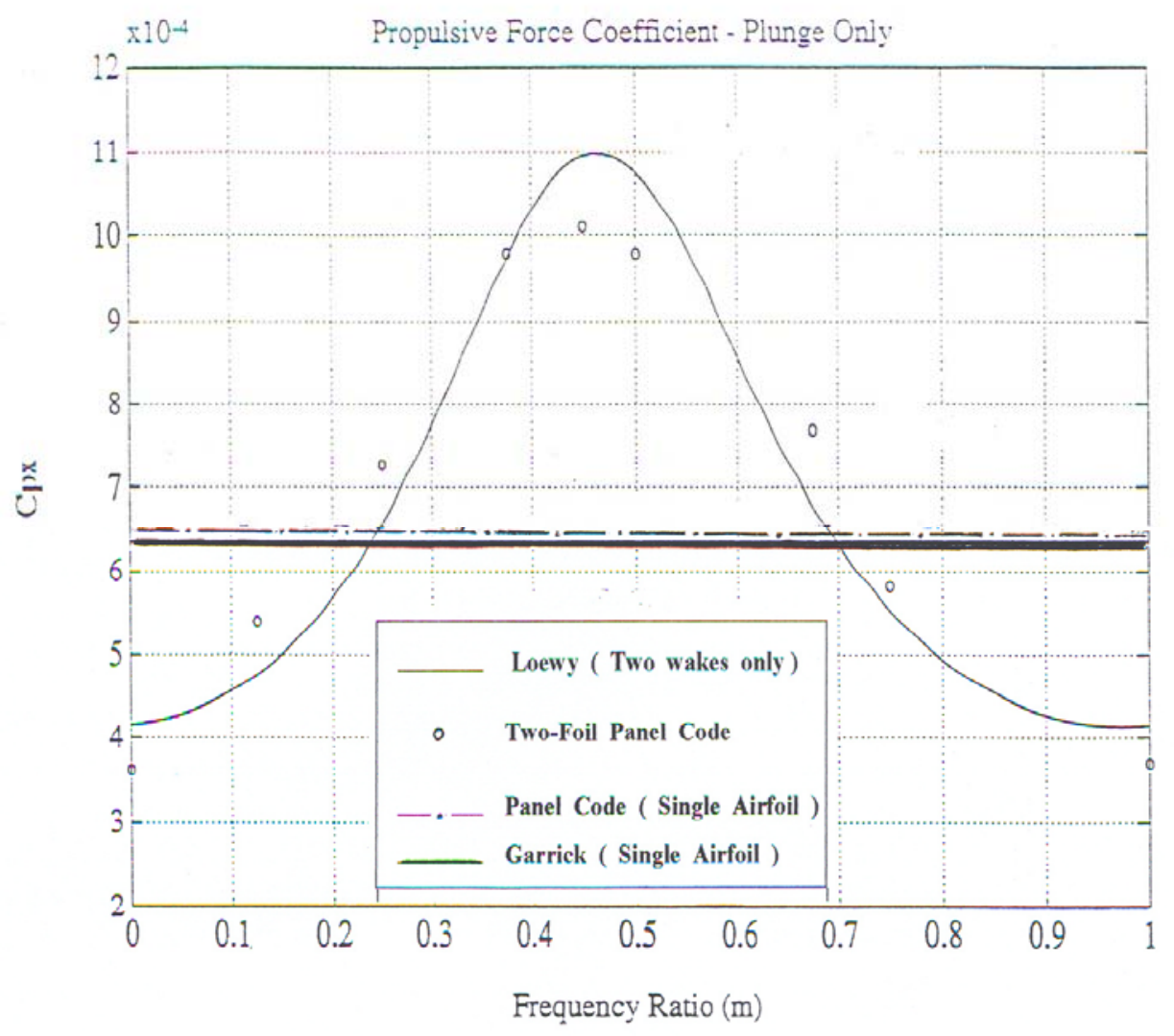

Fig.7 Average propulsive force due to plunge versus wake phasing, (m). 